\title{
The Coupled Brains of Captivated Audiences
}

\author{
An Investigation of the Collective Brain Dynamics \\ of an Audience Watching a Suspenseful Film
}

\author{
Ralf Schmälzle@e and Clare Grall
}

Department of Communication, Michigan State University, East Lansing, MI, USA

\begin{abstract}
Suspense not only creates a strong psychological tension within individuals, but it does so reliably across viewers who become collectively engaged with the story. Despite its prevalence in media psychology, limited work has examined suspense from a media neuroscience perspective, and thus the biological underpinnings of suspense remain unknown. Here we examine continuous brain responses of 494 viewers watching a suspenseful movie. To create a time-resolved measure of the degree to which a movie aligns audience-wide brain responses, we computed dynamic inter-subject correlations of functional magnetic resonance imaging (fMRI) time series among all viewers using sliding-window analysis. In parallel, we captured in-the-moment reports of suspense in an independent sample via continuous response measurement (CRM). We found that dynamic inter-subject correlations over the course of the movie tracked well with the reported suspense in the CRM sample, particularly in regions associated with emotional salience and higher cognitive processes. These results are compatible with theoretical views on motivated attention and psychological tension. The finding that fMRI-based audience response measurement relates to audience reports of suspense creates new opportunities for research on the mechanisms of suspense and other entertainment phenomena and has applied potential for measuring audience responses in a nonreactive and objective fashion.
\end{abstract}

Keywords: suspense, fMRI, inter-subject correlation, media neuroscience

Movies have powerful effects on audiences, and they effectively guide psychological processes and create strong experiences that evolve over time. When a movie is received by an audience, this leads to reactions and experiences that are shared across audience members. Perhaps the most salient example of this is the genre of suspense. Indeed, when different individuals watch a suspenseful movie, strong and collectively shared responses can be observed, and audience members report on similar experiences during specific story moments (Vorderer, Wulff, \& Friedrichsen, 2013) despite the many idiosyncratic differences that exist in individual experience.

The temporal and collective nature of suspense is implicitly acknowledged in media psychological theories, but limited previous research has honored these characteristics. Neuroimaging provides time-resolved information about regional brain responses while circumventing the need for interruption and thus can be used to study how movies align audiences as they unfold. This study investigates the relationship between aligned audience brain responses and audience perceptions in response to suspenseful content. We first provide a framework for understanding brain responses to movies over time and then discuss the phenomenon of suspense and its power in studying audience brain alignment. Lastly, we test this framework using data from audiences watching a suspenseful film.

\section{The Collective Nature of Continuous Audience Responses to Movies}

Watching a movie creates a myriad of microlevel media effects (Weber et al., 2015). The eyes and ears of each audience member will continuously respond to the stream of images and sounds, which are converted into neural signals and propagated to the visual and auditory regions of each viewer's brain. Studies on the mechanisms of vision and audition reveal the brain regions involved in elementary processes related to color vision, form, and object perception, as well as auditory processes such as pitch analysis and voice perception (Mesulam, 1998). Because the architecture of the visual and auditory systems is similar across all humans, watching the same movie will evoke similar functional responses across different viewers' brains. The varying onscreen images will elicit similar brain activity time series in the visual cortices of all viewers, and listening to the soundtrack will elicit similar processing in auditory regions. 
A.

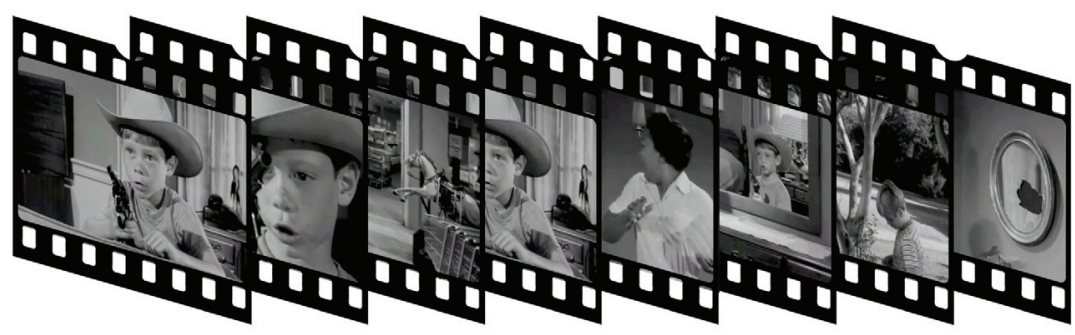

B.

STATIC ISC

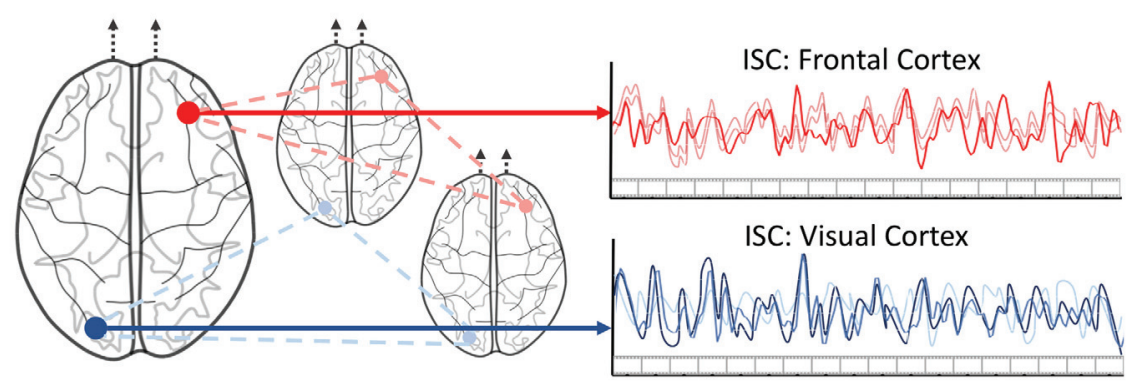

C.

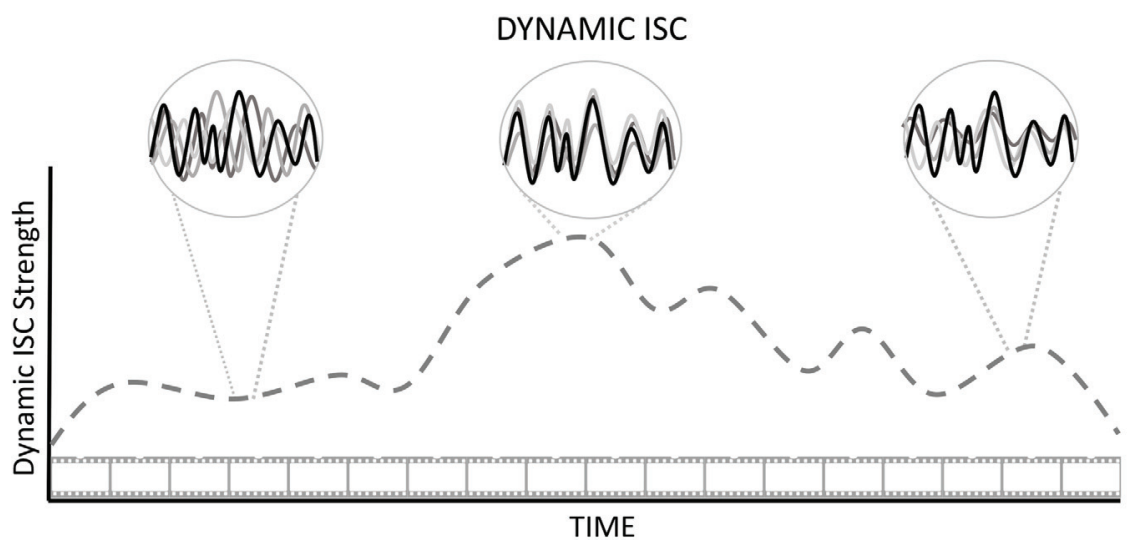

Figure 1. Theoretical rationale of static and dynamic inter-subject correlation analysis (ISC). (A) Brain activity time series are recorded from different recipients processing the same movie. In brief, the movie's story follows a young boy as he plays around his town with what he believes to be a toy gun. He encounters several close calls as he repeatedly pulls the trigger while pointing the gun at people. (B) The entire brain activity time series can be compared across viewers to assess the degree to which they become aligned during the reception process. (C) A dynamic version of ISC, computed over a moving time window, reveals how brain time series across audience members become more or less similar as the movie unfolds.

To measure these brain responses and compare their similarity between audience members, we can record their brain activity using functional magnetic resonance imaging (fMRI) while they watch movies and we can compute intersubject correlations (ISC). In ISC analysis, one calculates cross-recipient correlations between the brain activity time series measured in corresponding regions of different viewers' brains while they are exposed to the same message (Hasson et al., 2008; Hasson, Malach, \& Heeger, 2010; Schmälzle \& Grall, in press). As illustrated in Figure 1, fMRI time series from viewers' visual cortex can be extracted and compared to identify the degree to which they concur. By repeating this analysis for all viewers and across all brain regions, one can assess where in the brain and how strongly a given movie aligns neural processing across audiences. In this sense, ISC analysis yields an objective measure to examine collectively shared audience responses.

Importantly, when an audience watches a movie, the brain regions that exhibit such collectively shared processes extend beyond sensory regions and include postperceptual 
regions associated with various cognitive and affective functions. Decades of media psychology research demonstrate that movies do much more than only stimulate vision and audition. Instead, cognitive and emotional audience responses are key to their popularity (Tamborini, Bowman, Eden, Grizzard, \& Organ, 2010). For example, ISC in higher-order brain regions differs between listeners of the same story depending on whether they possess language knowledge and thus either can or cannot follow the content (Honey, Thompson, Lerner, \& Hasson, 2012). Furthermore, when people are given different background knowledge that suggests different interpretations of the same story, people with similar interpretations show more similarity in their brain responses (Yeshurun et al., 2017). Thus, rather than only reflecting common reactions driven by cuts, sounds, and image properties, assessing collective brain responses through ISC analysis can also aid the investigation of common psychological processes engaged by movies.

Mounting evidence suggests that ISC is sensitive to the strength of message content, be it in terms of strong verbal arguments or the potential to provoke strong affective responses, or due to the relevance that content has for receivers. Heightened ISC across audience brains has been shown during powerful political speeches, and health messages that were evaluated as more effective also prompt higher ISC than weaker messages, particularly in mediofrontal brain regions (Imhof, Schmälzle, Renner, \& Schupp, 2017; Schmälzle, Häcker, Honey, \& Hasson, 2015). Together with several other studies (Dmochowski et al., 2014; Nummenmaa, Lahnakoski, \& Glerean, 2018; Nummenmaa et al., 2014), this suggests that ISC analysis could be beneficial to investigate suspense, which has been intensively studied within media psychology (Vorderer et al., 2013; Zillmann, 1980) and has begun to attract interest from neuroscience (Bezdek et al., 2015; Lehne et al., 2015).

\section{Suspense: Theoretical Perspectives and Empirical Approaches}

Everyone who has seen a movie by Alfred Hitchcock knows how powerfully suspense can affect viewers. Suspense characterizes an affective state associated with conflict and uncertainty regarding an emotionally significant event that motivates future-oriented prediction and desire for a resolution (Lehne \& Koelsch, 2015). To illustrate this, consider one of Hitchcock's classic strategies, that is, to give the audience information that the characters lack, for example, by showing a villain hiding a bomb before the protagonists enter the scene. The audience's affection for the protagonists and the uncertainty regarding whether the bomb will go off create a mix between fear and hope and a motivational tension that keeps viewers focused on the unfolding story events. In this way, suspense taps into processes that cut across the domains of cognition and emotion, which are often described as a dichotomy but actually are intimately interwoven (Pessoa, 2013).

These powerful effects of suspense make it an ideal context for studying engaged audiences. As argued earlier, the fact that the architecture and function of visual and auditory sensory systems are conserved across humans explains why their brains will respond similarly to the same visual and auditory events. Critically, a parallel argument can be made for suspense in that it taps into deeply rooted motivational and social mechanisms, such as the predisposition to empathize with others and fear for their well-being (Frith \& Frith, 2012; Olsson, Nearing, \& Phelps, 2007; Tamborini, 2011). Therefore, just like processing sensory aspects of a continuous movie will prompt similar processing across viewers, we can also expect that the tension produced by suspense should induce similar brain responses, although this would likely occur in different regions that will be discussed later (Adolphs \& Anderson, 2018). However, in contrast to the sensory systems, the brain processes underlying suspense and other hot-cognition phenomena require more complicated conceptual content to become responsive. Movies are well-suited to advance this research because they can effectively evoke suspense in such a way that the time-locked content is identical in its presentation to all audience members, which in turn fulfills the prerequisite for ISC analysis to identify shared audience responses.

\section{The Interface Between Media, Attention, and Motivation}

It is clear that when a message can activate fundamental motivational systems that are shared across humans, this will affect how the message is encoded, processed, and stored (Fisher, Keene, Huskey, \& Weber, 2018). For instance, the LC4MP (Lang, 2009), which views message processing as a continuously evolving process and is compatible with cognitive neuroscience, emphasizes the importance of motivation in understanding cognitive effects. The LC4MP's conceptualization of motivation is based on the hypothesized dual motive systems in the brain, which organize appetitive and aversive behaviors (Cacioppo, Gardner, \& Berntson, 1999; Lang, 2010). In brief, real-life stimuli that carry affective significance can activate core motive circuits, although it must be noted that this does not necessarily map onto the activity of single brain regions, and the systems are complex in ways that simple labels such as appetitive and aversive tend to mask. Importantly, mediated representations, such as an image showing somebody being attacked, can also engage these systems.

Previous studies from affective neuroscience reveal a prioritized processing of emotional content, which has 
been interpreted as motivated attention (Schupp, Flaisch, Stockburger, \& Junghöfer, 2006; Schupp, Kirmse, Schmälzle, Flaisch, \& Renner, 2016). Regionally, these effects have not only been reported for affectivemotivational brain systems including mediofrontal regions, subcortical regions, and key hubs in the salience network such as the anterior cingulate cortex and the insula, but also as more distributed enhancements of sensory-perceptual processes (Pessoa, 2018; Schupp et al., 2007). Of note, attention here serves as an umbrella term for a collection of processes that can amplify or sustain responses for visual, auditory, visual, and higher-order representations (Chun, Golomb, \& Turk-Browne, 2011; Sara \& Bouret, 2012). The notion of motivated attention extends the cognitive notions of automatic/bottom-up or task-based/ top-down selective attention in a way that is very compatible with media psychological research and more naturalistic approaches to the phenomenon (Fawcett, Risko, \& Kingstone, 2015). Indeed, attention is naturally attracted to motivationally relevant stimuli, like when someone notices a murder on screen despite completing some separate task. However, it will be apparent to media scholars that work using static images and brief sounds to study the attention-motivation interface is less suited for narrative phenomena that build on continuous information. Moreover, although the motivated attention literature has demonstrated that motivationally relevant stimuli have very robust and thus intersubjectively consistent effects on brain activity, there has been no focus on audience-wide effects that are central to mass communication and media research.

\section{The Current Study and Hypothesis}

Suspense represents an inherently temporal experience that ebbs and flows throughout a movie as controlled by plot development (Zillmann, 1996). This necessitates a research approach that takes time into account, yet previous work has largely relied on static or retro- and introspective measures (Watt, 1994). The ISC approach has potential to unpack the reception process by providing spatially resolved and temporal information about how movies engage audiences at the neural level, yet theoretical connections with media psychology remain scarce (Dudai, 2012; Hasson et al., 2008; Schmälzle \& Grall, in press). Furthermore, previous work on audience-wide ISC in response to motivational messages has focused on comparing different messages but rarely examined dynamic fluctuations of ISC within a message, such as during the more suspenseful or less suspenseful parts of a movie. Finally, although each viewer responds to suspense individually, the key processes should unfold similarly across viewers and promote a shared experience.
On the basis of these considerations, we studied how suspense modulates audience brain responses and how these relate over time to reports of suspense in audiences at large. We used a large dataset in which fMRI had been recorded while participants viewed a short movie by Alfred Hitchcock (Shafto et al., 2014), for which we obtained reports of suspense via continuous response measurement from an independent audience (Biocca, David, \& West, 1994). We reasoned that if ISC reflects engagement with strong message content, and suspense similarly activates deeply rooted psychological processes across audience members (Lehne \& Koelsch, 2015), then fluctuations in ISC across a movie should be linked to variations of reported suspense. This logic is laid out in Figure 1C. Note that both dynamic ISC as well as the time-varying strength of suspense reports represent group-level metrics; dynamic ISC indexes variations in audience alignment as events unfold, and CRM reports of suspense index the strength of the audience-wide experience at specific moments in the movie.

Accordingly, we hypothesized that dynamic ISC of fMRI data will be associated with continuously reported suspense (H1). We first focused on ISC dynamics throughout the brain (H1a) because the notion of motivated attention, which would comprise visual, auditory, and higher-conceptual activities to process the content, should be reflected in distributed ISC effects. Second, based on work on motivational brain systems, we expected that regions of the salience network, key in attentional prioritization and emotional processes (Menon, 2015; Roy, Shohamy, \& Wager, 2012), would exhibit a particularly tight relationship between dynamic ISC and suspense reports at the level of aggregate audiences (H1b).

\section{Method}

\section{Sample and Procedure}

\section{Neuroimaging Sample}

We analyzed an open dataset acquired by the Cambridge Center for Aging and Neuroscience (Cam-CAN), an initiative focused on age-related brain development (Campbell et al., 2015; Shafto et al., 2014). The final sample for fMRI time series analysis included 494 participants ( 252 female). Details on the participant selection can be found in the Cam-CAN data descriptor (Shafto et al., 2014). Eligibility measures included an English-language requirement and meeting criteria for fMRI scanning.

\section{Continuous Response Measurement Sample}

A total of 22 human raters were recruited to provide continuous response measures (CRM; Biocca et al., 1994; Kempter \& Bente, 2004). While viewing the same movie 
as the fMRI sample, participants were directed to "continuously evaluate the degree of suspense" they were experiencing using a mouse to direct an on-screen slider (scale ranging from $0=$ low suspense to $100=$ high suspense, starting at 50; sampling rate $5 \mathrm{~Hz}$; Nummenmaa et al., 2012).

\section{Movie}

The movie stimulus is an episode titled "Bang! You're Dead" from the television show Alfred Hitchcock Presents. The story follows a young boy as he plays around town with what he believes to be a toy gun. He encounters several close calls as he repeatedly pulls the trigger while pointing the gun at people. This narrative is ideally suited to evoke suspense because the audience is knowledgeable that the gun is real and loaded with one bullet, but the boy and potential victims are unaware. The episode had an original run-time of $30 \mathrm{~min}$ and was edited down to $8 \mathrm{~min}$ while maintaining the plot.

\section{fMRI Acquisition and Processing}

MRI data were acquired on a 3-T Siemens TIM Trio System with a 32-channel head coil. Functional images were recorded during movie viewing (using a multi-echo T2-weighted EPI sequence, TR $=2,470 \mathrm{~ms}, 32$ axial slices, voxel size $=3 \mathrm{~mm} \times 3 \mathrm{~mm} \times 4.44 \mathrm{~mm}$, 193 volumes), and structural scans were acquired using a MPRAGE sequence. For details, see the report on the CamCan study (Shafto et al., 2014).

The imaging data were preprocessed using the nipype framework (Gorgolewski et al., 2011), including motion correction, slice-time correction, co-registration, and nonlinear normalization to the MNI standard. Functional data were detrended and high-pass filtered at $0.01 \mathrm{~Hz}$, and time series were extracted using NiLearn (Abraham et al., 2014). From the original dataset of 646 participants, data were excluded if they were incomplete, exhibited abnormalities during anatomical normalization or technical failures, or contained more than $1.5 \mathrm{~mm}$ of movement. This resulted in a final sample of 494 participants.

\section{Inter-Subject Correlation (ISC) Analysis}

The analysis was carried out on the brain activity time series extracted from the Shen Parcellation (Shen, Tokoglu, Papademetris, \& Constable, 2013), which consists of 268 regions from the entire brain (see Figure 2). From each of these regions, time series were extracted resulting in a matrix of 193 TRs $\times 268$ regions $\times 494$ viewers. This matrix was submitted to ISC analysis (Hasson et al., 2010; Hasson, Nir, Levy, Fuhrmann, \& Malach, 2004). In brief, ISC assesses the correlations between fMRI time series from corresponding brain regions across different individuals exposed to the same stimulus (see Figure 1).

\section{Static ISC}

First, to demonstrate that the movie aligns audience brain responses and in which areas this happens throughout the entire movie, we conducted an ISC analysis using the time series form the whole movie, termed static ISC. To test the statistical significance of this static ISC, we conducted permutation tests that use phase randomization to generate surrogate time series with random temporal alignment across viewers (see Nastase, Gazzola, Hasson, \& Keysers, 2019 or Schmälzle, Imhof, Grall, Flaisch, \& Schupp, 2017 for an introduction). Results were statistically significant $(p<.01)$ after correction for multiple comparisons (FDR; Benjamini \& Yekutieli, 2001).

\section{Dynamic ISC}

Previous ISC research has mainly focused on static ISC, which summarizes the similarity of brain activity during the entire movie. To create a time-resolved measure of the degree to which audience-wide brain responses become aligned, we computed a dynamic ISC analysis using a sliding-window correlation approach (Preti, Bolton, \& Van De Ville, 2017). Specifically, for each region, we computed ISC analysis using data from a window with a width of 15 TRs (about 37 s). By sliding the centered window over the time series (shifted successively with an increment of $1 \mathrm{TR}$ ) and keeping track of the resultant ISC, we created a measure of cross-receiver brain similarity over time called dynamic ISC (see Figure 1C).

\section{CRM Data Collection and Analysis}

CRM data were collected over the Internet using a javascript-based tool that showed the movie and collected slider ratings from viewers (Nummenmaa et al., 2012). The data were processed using python and averaged across individual traces to create an aggregate measure of suspense over time, as is common in CRM analysis (Biocca et al., 1994; Kempter \& Bente, 2004). Finally, the averaged CRM traces were down-sampled from 5 to $0.4049 \mathrm{~Hz}$ to match the resolution of the fMRI data. Split-half analyses revealed that the results were highly robust (mean $r=$ 0.87 across 1,000 permutations).

All analyses are documented at https:/github.com/ nomcomm/CamCanSuspenseISC_JMP.

\section{Results}

\section{Shared Brain Responses During Movie Viewing: Static ISC}

Figure 2 shows the results of the ISC analysis computed across the entire movie. As can be seen, movie viewing 


\section{A. STATIC ISC: AUDIENCE BRAIN ALIGNMENT DURING MOVIE-VIEWING}

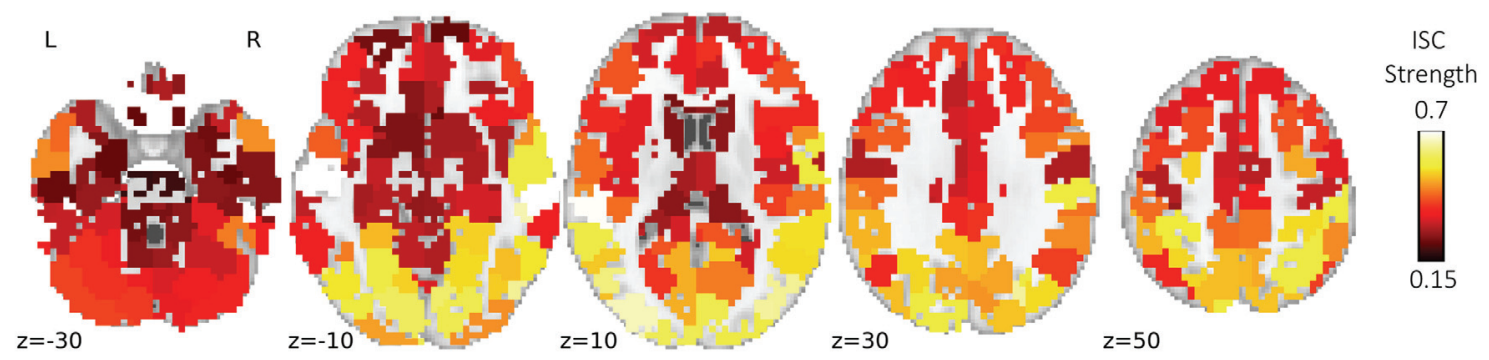

B.

PARIETAL REGION ACTIVITY BY SUBGROUP

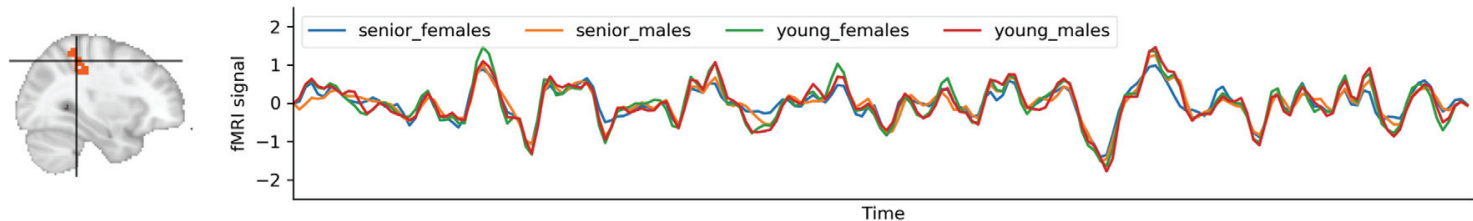

C.

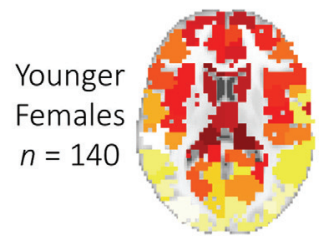

ISC BY SUBGROUP

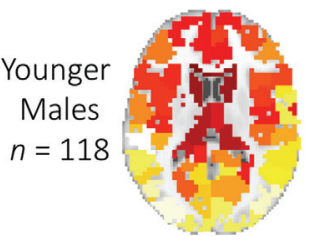

D. NO ISC DURING REST

Figure 2. Static ISC results. (A) ISC results computed for the entire movie (static ISC). As expected, highest ISC emerges for visual and auditory regions, but effects are present throughout the brain. Please note that contrary to conventional fMRI activation maps, these maps represent similar processing across the brains of the audience of 494 viewers. (B) Example time series from a region in the parietal cortex, averaged by age and gender groups to reveal the similar processes in this region. (C) Static ISC results by subgroups (same color scale in A). (D) When the participants only lie in the scanner and there is no common movie, brain activity is not correlated and the regional time series (same region as in B) average out to a flat line. ISC = inter-subject correlations.

evokes shared brain responses (ISC). As expected, the similarity of brain activity time series across viewers is strongest in visual and auditory regions with lesser, but significant, correlations in frontal regions $(p<0.01$, FDR corrected). The spatial distribution of inter-subject similarity matches with previous reports (Hasson et al., 2004; Lahnakoski et al., 2014; Schmälzle, Häcker, Renner, Honey, \& Schupp, 2013) showing that ISC extends into higher-order regions involved in social cognition, salience processing, and executive control. Very similar results emerge when the same analysis is carried out on data from subgroups, such as for younger and older females or males (Figure 2), and the spatial pattern of results is highly similar in each subgroup (spatial correlations all $r>0.96$, $p<0.01$.

We also conducted ISC analyses on resting-state data in which participants did not view any common stimulus. This analysis confirmed that without the movie acting as an "audience-aligner," the different brains exhibit no similar processes but idle at their own pace (Figure 2D). 

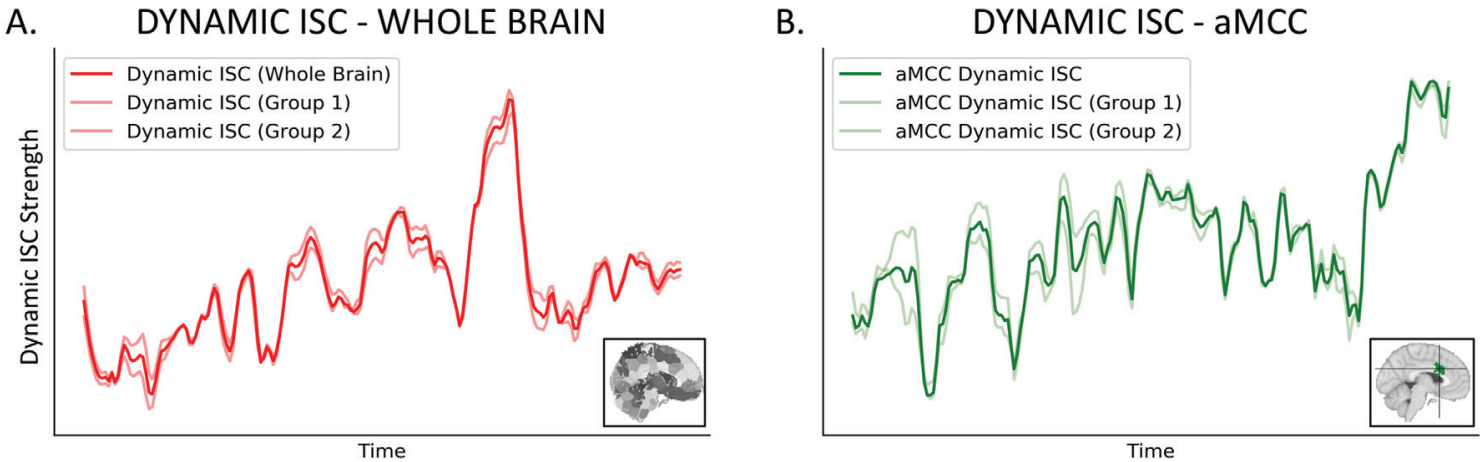

Figure 3. Dynamic ISC results. (A) Dynamic ISC averaged across all regions. (B) Dynamic ISC for the anterior cingulate cortex (ACC). In both panels, the shaded lines represent the dynamic ISC results when computed across two random halves of the full dataset, demonstrating the high stability of results. ISC = inter-subject correlations.

\section{Collective Audience Brain Alignment Over Time: Dynamic ISC}

Next, to generate a time-resolved measure of how well the audience's brain time series become more or less aligned as the movie unfolds, we computed dynamic ISC analysis. Specifically, for each region, we computed an ISC analysis within a given time window, recorded each result, and repeated the procedure with a shifted window. The results of this analysis are illustrated in Figure 3. Figure 3A shows the evolution of dynamic ISC averaged across the brain and Figure $3 \mathrm{~B}$ focuses on the anterior cingulate cortex, a key node of the salience network.

To demonstrate the stability of this measure, we repeated the analysis for independent subgroups of the 494-person audience. Specifically, we randomly divided the audience into halves and ran dynamic ISCs for each. As shown by the shaded lines in Figure 3, the independent assessments confirm the stability of the results (whole brain: $r=0.9$, $p=4.33 \mathrm{e}-111$; ACC: $r=0.85 ; p=2.32 \mathrm{e}-57)$. We also repeated this computationally intensive procedure via permutations (see supplementary analyses in the reproducibility package: https://github.com/nomcomm/CamCanSuspenseISC_ JMP). All analyses confirmed the robustness of the dynamic ISC.

\section{Ratings of Suspense (CRM)}

To capture the degree of reported suspense over time, the continuous ratings of suspense were averaged across the group (Figure 4). Reported suspense varies over time, as shown by the $95 \%$ confidence interval around the mean for each timepoint. Combined visual inspection of the movie and reported suspense confirmed that the latter mirrors the narrative structure of the movie. For instance, peaks in reported suspense coincide with moments depicting imminent danger to innocent characters.
CONTINUOUS RATINGS OF SUSPENSE

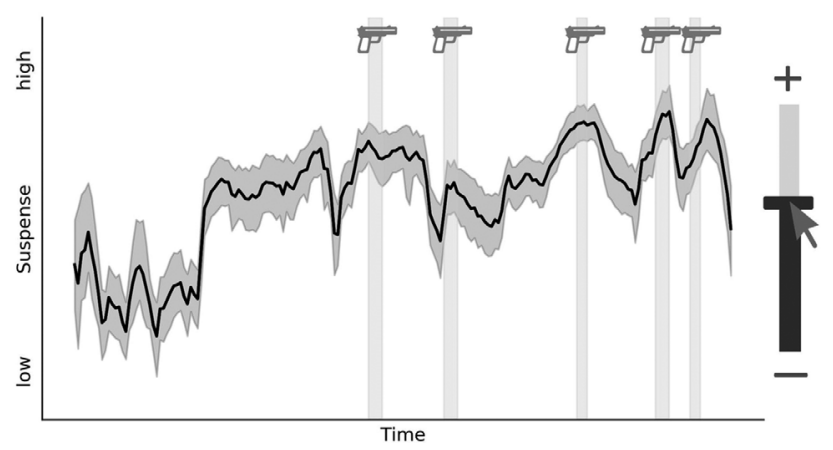

Figure 4. Continuous reports of suspense. The group-averaged time series of continuous ratings of suspense. The shaded area around the time series represents the 95\% confidence interval around the mean at each time point. The gray-shaded vertical blocks indicate movie scenes comprising close-ups of the gun when the boy has it aimed at a person.

\section{Relationship Between Dynamic ISC and Reported Suspense}

The dynamic ISC analysis yields a time-resolved measure of the degree of collective brain similarity, which we theorized would respond to content-driven elements that promote engagement with the narrative. The CRM captures the extent to which an independent audience reports suspense over time, and we hypothesized that this should track with the collective alignment of brain responses. Supporting our prediction, fluctuations in dynamic ISC strength between the viewers whose brains are scanned are related to reports of experienced suspense in an independent audience, as indexed via cross-correlations. As seen in Figure $5 \mathrm{~A}$, this is the case for the whole-brain dynamic ISC; the dynamic ISC measure (averaged across the entire brain) takes a similar overall trajectory to the reported suspense $(r=0.47, p=4.54 \mathrm{e}-12)$. 
A. WHOLE BRAIN DYNAMIC ISC $\rightarrow$ SUSPENSE CRM

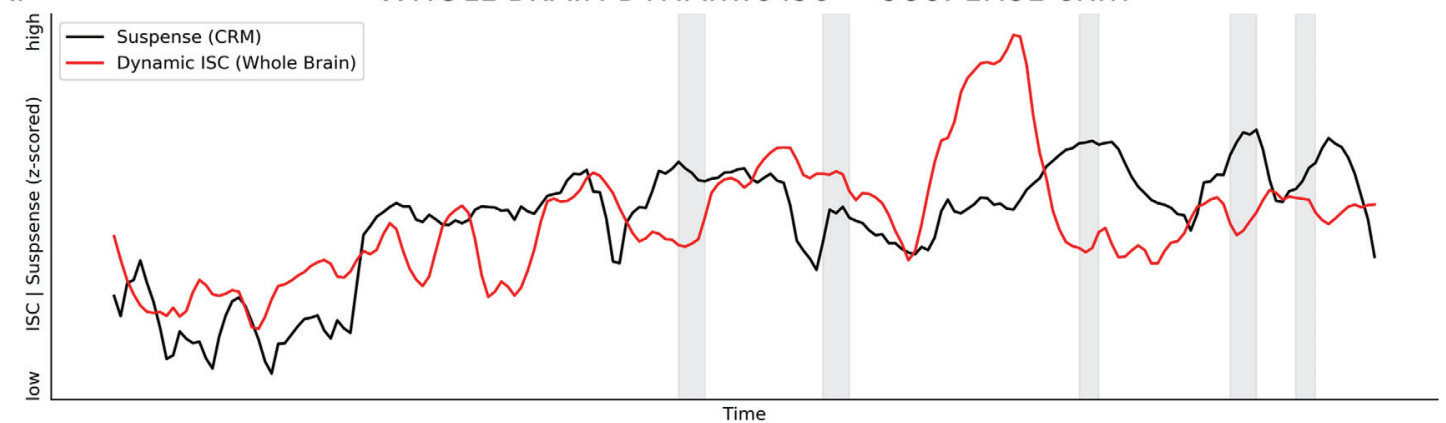

B.

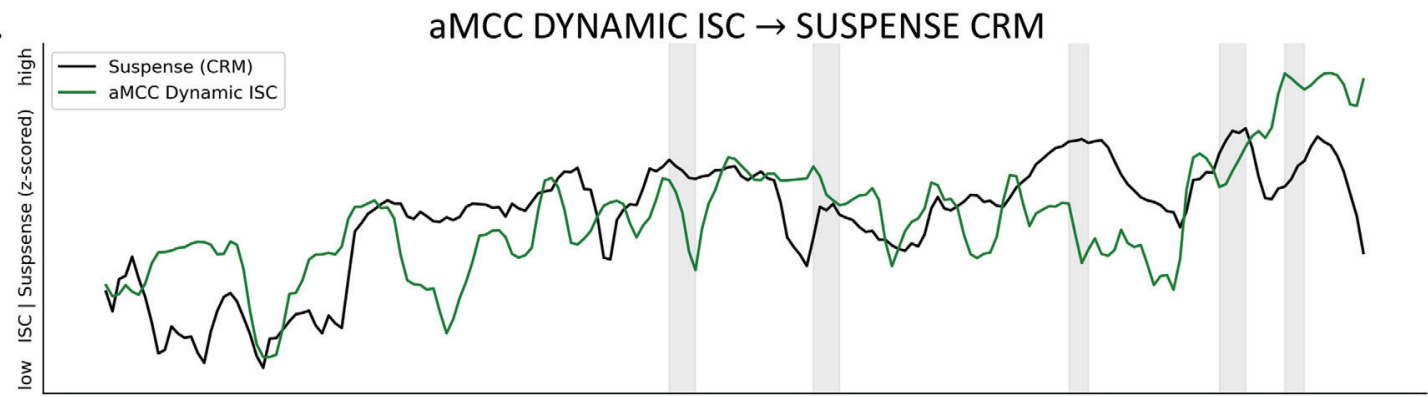

C.

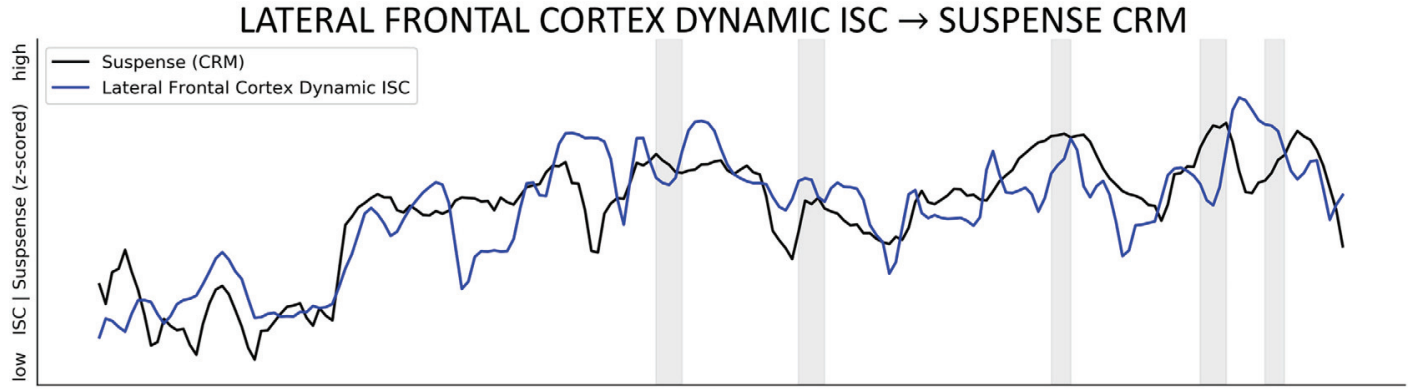

D.

\section{DYNAMIC ISC $\rightarrow$ SUSPENSE CRM ACROSS REGIONS}
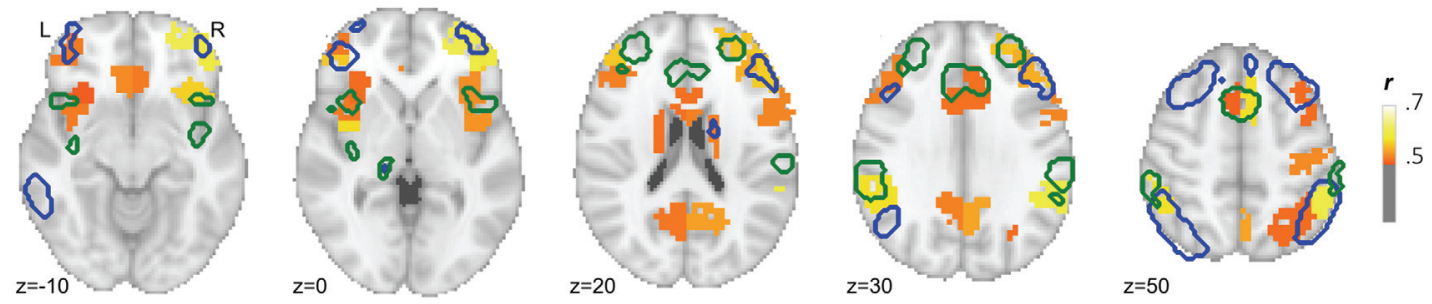

Figure 5. Relationship between dynamic ISC and reported suspense. (A) Fluctuations of dynamic ISC relate to reports of suspense in an independent audience. The black line represents the average suspense CRM (see Figure 4). Moments when the movie shows a close-up of the gun as the boy aims at a potential victim and fingers the trigger are shaded in gray. The $y$-axis represents the strength of dynamic ISC and the extent of reported suspense, respectively (both standardized). (B) Same analysis for the anterior midcingulate cortex (aMCC), a key node in the salience network. (C) Same analysis for lateral frontal cortex. (D) Correlation between all individual regions' ISC trajectories and reported suspense, thresholded at $r>$.5. Regions in which ISC tracks most closely with suspense map well onto the saliency (outlined in green) and the executive control (outlined in blue) networks (Shirer, Ryali, Rykhlevskaia, Menon, \& Greicius, 2012). CRM = continuous response measurement; ISC = intersubject correlations.

Whereas Figure 5A represents the dynamic ISC result averaged across the entire brain, regional analyses of dynamic ISC point to some specificity of this effect (Figures 5B-5D). The highest correlations between dynamic ISC and suspense ratings were observed for the midcingulate gyrus $(r=0.70, p=2.21 \mathrm{e}-30)$, the angular gyrus $(r=0.71, p=6.83 \mathrm{e}-31)$, and the right lateral prefrontal cortex $(r=0.79, p=1.22 \mathrm{e}-42)$. Correlations between $0.5<r<0.7$ $(p<0.01)$ emerged in the anterior cingulate, the bilateral anterior insulae, bilateral frontal cortices, the precuneus, medial prefrontal cortex, and the bilateral angular gyri. These regions are prominent parts of higher-order brain 
systems such as the saliency and executive networks, which are indicated by the green and blue outlines in Figure 5D, respectively. We confirmed that the reported relationship between dynamic ISC and reported suspense holds after regressing out the potential influence of motion (for both the results across the brain as well as for the regional analyses). We also confirmed these relationships in subsamples of test audiences with varying amounts of motion.

Of note, there are also regions in which dynamic ISC and reported suspense do not track with each other. These include the majority of the visual and auditory systems. Closer inspection revealed that these regions do not just exhibit a fixed level of dynamic ISC throughout the movie, but they show fluctuations over time. However, in this movie, these fluctuations in audience brain alignment do not correlate with the reported suspense experiences.

\section{Discussion}

This study examined how movies align audience brains, specifically how people "tune in" more to the movie during moments of peak suspense or motivational tension (Lehne \& Koelsch, 2015), which should result in more tightly aligned neural responses (Schmälzle \& Grall, in press). Our hypothesis stated that fluctuations in the degree to which audience brain responses become collectively aligned during the movie should be related to the strength of reported suspense in independent viewers. The results support this prediction (Figure 5).

Looking at dynamic ISC by region, we find that regions showing a particularly close relationship to suspense are those that belong to the salience and executive networks (green and blue outlines in Figure 5D). The salience network has often been linked to the integration of sensory, emotional, and cognitive information, and particularly to processes related to anxious apprehension and anticipatory anxiety (Etkin, Egner, \& Kalisch, 2011; Menon, 2015). Additionally, the executive network, which encompasses the frontoparietal cortex, is involved in higher cognitive functions related to control and executive attention (Pessoa, 2009; Seeley et al., 2007). It makes sense that these regions would become particularly engaged across viewers as they tune in to the suspense movie based on the high relevance of unfolding events (Schmitz \& Johnson, 2007; Sherif \& Sargent, 1947). Although these types of processes are certainly compatible with those that would become engaged by suspense, it is important to note that most prior neuroimaging studies were using different methods, and the limits of reverse inference must be acknowledged (Poldrack, 2006).

Two immediate areas for expanding this research are the paradox of suspense and the cascading of affective responses. The paradox of suspense refers to the phenomenon that movies remain suspenseful even when the outcome is known (Carroll, 2013). Thus, even when objective uncertainty no longer exists, people respond as if it were still present, at least to some degree. By examining neural changes across repeated viewings of the same movie (Schmälzle et al., 2017), researchers could elucidate the basis of this phenomenon and related effects of narratives on socio-affective processing. A second avenue for research would be to zoom in on the temporal orchestration of cognitive predictions and affective anticipations that promote the mix of negatively valenced fear and positively valenced hope that characterize suspense. Future studies could focus on the micro-mechanisms underlying mounting anxious apprehension (Najafi, Kinnison, \& Pessoa, 2017) or the onset of relief when suspense gets defused. Both prediction and anticipation have been studied in the cognitive and affective neurosciences (Berridge, 2019; Friston, 2010; Knutson \& Greer, 2008) and the LC4MP emphasizes the role of co-activation (Fisher et al., 2018), suggesting that these areas are ripe for theoretical integration. Third, although dynamic ISC in sensory cortices did not correlate with the trajectory of collective suspense reports in this movie, future work could examine whether other psychological processes or content features exhibit relationships to brain activity (cf. Raz \& Hendler, 2014). The free availability of this and other large datasets (Vanderwal, Eilbott, \& Castellanos, 2018), theoretical and methodological advances in specifying relevant processes (Fisher et al., 2018; McNamara, De la Vega, \& Yarkoni, 2017), and the provisioning of our analysis code greatly facilitates such efforts.

\section{Integration Between Media Psychology and Neuroscience}

The notion that motivated processing of conceptual content is central to media entertainment and strongly modulates cognitive responses has a long history in media psychology, as perhaps most prominently articulated by the LC4MP (Fisher et al., 2018; Lang, 2009). However, testing and advancing these ideas requires zooming in on relevant processes over time with a focus on motivation and affect, which are notoriously difficult to study despite being key modulators of executive processes. It is clear that neuroimaging has potential to advance this research, but prior work in cognitive neuroscience has predominantly focused on abstract cognitive processing and laboratory tasks that bear little resemblance to the powerful effects of media, particularly suspense. The field of affective neuroscience, in turn, has examined how affective images prompt motivated attention, but this work mainly employed static images with explicit perceptual but no narrative content 
(e.g., depictions of mutilations and erotica), which barely engage higher-order cognitive processing (Bradley, 2009; Lang, Bradley, \& Cuthbert, 1997). The current study combines these lines of research in a mutually beneficial way; by using a conceptually powerful stimulus, we can engage higher-order systems involved in narrative processing, social cognition, and emotion in a very controlled manner to study their effects on individual brain activity and whole audiences. Although more work is needed, this approach is suited to study the audience response to dynamic media from an objective, neural perspective, and to link neural effects to content and subsequent responses (Dudai, 2012; Hasson et al., 2008; Schmälzle \& Grall, in press).

\section{Possible Generalization to Other Media Phenomena}

A follow-up question is whether this approach can be generalized to other phenomena and viewing experiences. In terms of content types and genres, the argument we made for suspense as an "audience-aligner" could also be made for horror, comedy, tragedy, and action. For instance, a scene depicting a character's sudden death is likely to prompt common responses across viewers no matter the genre. However, movies from different genres will likely recruit a mix of distinct and common brain systems to process the content and implement the motivational response. Our theorizing focuses on the strong audience response to motivationally relevant messages. This approach, to capture the "strength of the grip" (Hasson et al., 2008) a movie has on an audience, can be used across genres and even beyond movie viewing, such as effective speaking and health communication (Imhof et al., 2017; Schmälzle et al., 2015). Translational research has already begun to link neural data to outcomes such as memory, persuasion, and commercial success (Barnett \& Cerf, 2017; Chen et al., 2017; Dmochowski et al., 2014; Ki, Kelly, \& Parra, 2016; Pei, Schmälzle, Kranzler, O’Donnell, \& Falk, 2019).

\section{Strengths and Limitations}

This study advances a novel quantitative way to link collective brain responses to media with audience effects, as measured via CRM, extending the seminal work by Lazarsfeld and Stanton on the Program Analyzer into the domain of neuroscientific response measurement (Levy, 1982). This general framework can easily be adapted to other movie genres, to other media types such as spoken or written narratives and music, and also to link audience brain effects back to their elicitors in media content. An additional strength of this study is the large brain imaging sample, which provides robust aggregate measures (Turner, Paul, Miller, \& Barbey, 2018).

However, several limitations need to be acknowledged as well. With fMRI measures it is always important to consider subject motion (Power, Schlaggar, \& Petersen, 2015), although it is not clear a priori how motion relates to suspense, as it might either cause people to move less owing to absorption or more owing to agitation during a particular scene. Empirically, we find that the relationship between dynamic ISC and suspense holds when controlling for the potential influence of motion, which bolsters our confidence in the data and suggests that synchronous movement does not account for these effects.

It should be noted that the "Bang! You're Dead" episode has been used in previous studies (Dmochowski, Sajda, Dias, \& Parra, 2012; Hasson et al., 2008; Naci, Cusack, Anello, \& Owen, 2014), and some reported results are compatible with the notion that the degree of audience brain coupling tracks with reported suspense. The most closely related study is one that links time-resolved EEG-ISC to what is qualitatively described as "emotionally laden" moments (Dmochowski et al., 2012). However, this study used a slightly different version of the movie and a smaller audience. Similar findings have also been reported recently by the CamCAN team itself (Geerligs, Cam-CAN, \& Campbell, 2018), who discuss high ISC in relation to "salient events" as defined by qualitative inspection of content. This study uses the same stimulus and dataset but focuses primarily on how age relates to idiosyncratic responding without considering the topic of suspense from a media psychological perspective. Overall, these prior results bolster our interpretation and suggest that the integration of multimodal measures with fine-grained analyses of movie content and experience sampling methods is promising and, in fact, already ongoing (Haufe et al., 2018).

The short duration of the movie in combination with the low temporal resolution of fMRI is a limitation. Although the movie is clearly long enough to present an engaging story, the slow nature of fMRI measurement poses an upper limit for our ability to resolve effects in time. Furthermore, time series analyses often face the issues of nonstationarity and a lack of independence between samples, and these issues become more acute when limited data are available. Conventional approaches to control for these issues are insufficient as they run the risk of interfering with the phenomenon of study. We thus opted to stay close to the measured data and refrain from statistical transformations, but these issues should be kept in mind when interpreting the findings. We also note that future analyses might modify inevitable choices we had to make, such as the window size for dynamic ISC analyses or potential lags regarding the temporal alignment of neural and subjective data. Solutions for overcoming these issues include collecting more data 
and employing other measures such as EEG (Imhof, Schmälzle, Renner, \& Schupp, 2020). Fortunately, several large fMRI initiatives are currently scanning audiences while they are exposed to longer and more varied content (Dubois \& Adolphs, 2016; Richardson, Lisandrelli, Riobueno-Naylor, \& Saxe, 2018). The move toward large and freely available neuroimaging datasets including movie stimuli will make fMRI data easier to obtain for media scholars and create unique opportunities to expand the current research and address novel questions (Vanderwal et al., 2019). Going forward, it will also be important to integrate these databases with other methods, such as psychophysiology, to triangulate findings (Potter \& Bolls, 2012).

\section{Conclusion}

To conclude, this study examined how a suspenseful movie evokes similar responses in the brains of audience members. We found that the strength of audience-wide brain similarity varied over time and tracked with reports of suspense. These findings contribute to our understanding of the mechanisms of suspense in the message reception process from content, to audience brains, to subsequent effects on subjective reports.

\section{Research Transparency Statement}

Data used in this work were obtained from the CamCAN repository available at http://www.mrc-cbu.cam.ac.uk/ datasets/camcan (Shafto et al., 2014; Taylor et al., 2017). Code to reproduce and document the analyses is accessible online at https://github.com/nomcomm/ CamCanSuspenseISC_ JMP.

\section{References}

Abraham, A., Pedregosa, F., Eickenberg, M., Gervais, P., Mueller, A., Kossaifi, J., .. Varoquaux, G. (2014). Machine learning for neuroimaging with scikit-learn. Frontiers in Neuroinformatics, 8, 14.

Adolphs, R., \& Anderson, D. (2018). The neuroscience of emotion. Princeton, NJ: Princeton University Press.

Barnett, S. B., \& Cerf, M. (2017). A ticket for your thoughts: Method for predicting content recall and sales using neural similarity of moviegoers. The Journal of Consumer Research, 44, 160-181.

Benjamini, Y., \& Yekutieli, D. (2001). The control of the false discovery rate in multiple testing under dependency. The Annals of Statistics, 29, 1165-1188. https://doi.org/10.1214/ aos/1013699998

Berridge, K. C. (2019). Affective valence in the brain: Modules or modes? Nature Reviews. Neuroscience, 20, 225-234. https:// doi.org/10.1038/s41583-019-0122-8

Bezdek, M. A., Gerrig, R. J., Wenzel, W. G., Shin, J., Revill, K. P., \& Schumacher, E. H. (2015). Neural evidence that suspense narrows attentional focus. Neuroscience, 303, 338-345.

Biocca, F., David, P., \& West, M. (1994). Continuous response measurement (CRM): A computerized tool for research on the cognitive processing of communication messages. In A. Lang (Ed.), Measuring psychological responses to media messages (pp. 15-64). Mahwah, NJ: Erlbaum.

Bradley, M. M. (2009). Natural selective attention: Orienting and emotion. Psychophysiology, 46, 1-11.

Cacioppo, J. T., Gardner, W. L., \& Berntson, G. G. (1999). The affect system has parallel and integrative processing components: Form follows function. Journal of Personality and Social Psychology, 76, 839-855.

Campbell, K. L., Shafto, M. A., Wright, P., Tsvetanov, K. A., Geerligs, L., Cusack, R., ... Tyler, L. K. (2015). Idiosyncratic responding during movie-watching predicted by age differences in attentional control. Neurobiology of Aging, 36, 30453055. https://doi.org/10.1016/j.neurobiolaging.2015.07.028

Carroll, N. (2013). The paradox of suspense. In P. Vorderer, H. J. Wulff, \& M. Friedrichsen (Eds.), Suspense: Conceptualizations, theoretical analyses, and empirical explorations (pp. 81-102). Mahwah, NJ: Routledge.

Chen, J., Leong, Y. C., Honey, C. J., Yong, C. H., Norman, K. A., \& Hasson, U. (2017). Shared memories reveal shared structure in neural activity across individuals. Nature Neuroscience, 20, 115-125.

Chun, M. M., Golomb, J. D., \& Turk-Browne, N. B. (2011). A taxonomy of external and internal attention. Annual Review of Psychology, 62, 73-101.

Dmochowski, J. P., Bezdek, M. A., Abelson, B. P., Johnson, J. S., Schumacher, E. H., \& Parra, L. C. (2014). Audience preferences are predicted by temporal reliability of neural processing. Nature Communications, 5, 4567.

Dmochowski, J. P., Sajda, P., Dias, J., \& Parra, L. C. (2012). Correlated components of ongoing EEG point to emotionally laden attention - A possible marker of engagement? Frontiers in Human Neuroscience, 6. https://doi.org/10.3389/fnhum.2012.00112

Dubois, J., \& Adolphs, R. (2016). Building a science of individual differences from fMRI. Trends in Cognitive Sciences, 20, 425-443.

Dudai, Y. (2012). The cinema-cognition dialogue: A match made in brain. Frontiers in Human Neuroscience, 6, 248.

Etkin, A., Egner, T., \& Kalisch, R. (2011). Emotional processing in anterior cingulate and medial prefrontal cortex. Trends in Cognitive Sciences, 15, 85-93.

Fawcett, J., Risko, E., \& Kingstone, A. (Eds.). (2015). The handbook of attention. Cambridge, MA: MIT Press.

Fisher, J. T., Keene, J. R., Huskey, R., \& Weber, R. (2018). The limited capacity model of motivated mediated message processing: Taking stock of the past. Annals of the International Communication Association, 42, 270-290.

Friston, K. (2010). The free-energy principle: A unified brain theory? Nature Reviews Neuroscience, 11, 127-138.

Frith, C. D., \& Frith, U. (2012). Mechanisms of social cognition. Annual Review of Psychology, 63, 287-313.

Geerligs, L., Cam-CAN.\& Campbell, K. L. (2018). Age-related differences in information processing during movie watching. Neurobiology of Aging, 72, 106-120.

Gorgolewski, K., Burns, C., Madison, C., Clark, D., Halchenko, Y., Waskom, M., \& Ghosh, S. (2011). Nipype: A flexible, lightweight and extensible neuroimaging data processing framework in python. Frontiers in Neuroinformatics, 5, 13.

Hasson, U., Landesman, O., Knappmeyer, B., Vallines, I., Rubin, N., \& Heeger, D. J. (2008). Neurocinematics: The neuroscience of film. Projections, 2, 1-26.

Hasson, U., Malach, R., \& Heeger, D. J. (2010). Reliability of cortical activity during natural stimulation. Trends in Cognitive Sciences, 14, 40-48. https://doi.org/10.1016/j.tics.2009.10.011

Hasson, U., Nir, Y., Levy, I., Fuhrmann, G., \& Malach, R. (2004). Intersubject synchronization of cortical activity during natural vision. Science, 303, 1634-1640. 
Haufe, S., DeGuzman, P., Henin, S., Arcaro, M., Honey, C. J., Hasson, U., \& Parra, L. C. (2018). Elucidating relations between fMRI, ECoG and EEG through a common natural stimulus. Neurolmage, 179, 79-91. https://doi.org/10.1016/j.neuroimage. 2018.06.016

Honey, C. J., Thompson, C. R., Lerner, Y., \& Hasson, U. (2012). Not lost in translation: Neural responses shared across languages. The Journal of Neuroscience, 32, 15277-15283.

Imhof, M. A., Schmälzle, R., Renner, B., \& Schupp, H. T. (2017). How real-life health messages engage our brains: Shared processing of effective anti-alcohol videos. Social Cognitive and Affective Neuroscience, 12, 1188-1196.

Imhof, M., Schmälzle, R., Renner, B., \& Schupp, H. T. (2020). Strong health messages increase audience brain coupling. Neurolmage, 116527. https://doi.org/10.1016/j.neuroimage.2020.116527

Kempter, G., \& Bente, G. (2004). Psychophysiologische Wirkungsforschung: Grundlagen und Anwendungen [Psychophysiological Media Effects Research: Foundations and Applications]. In P. Mangold, G. Vorderer, \& G. Bente (Eds.), Lehrbuch der Medienpsychologie (1st ed.). Göttingen, Germany: Hogrefe.

Ki, J. J., Kelly, S. P., \& Parra, L. C. (2016). Attention strongly modulates reliability of neural responses to naturalistic narrative stimuli. The Journal of Neuroscience, 36, 3092-3101.

Knutson, B., \& Greer, S. M. (2008). Anticipatory affect: Neural correlates and consequences for choice. Philosophical Transactions of the Royal Society B: Biological Sciences, 363, 37713786.

Lahnakoski, J. M., Glerean, E., Jääskeläinen, I. P., Hyönä, J., Hari, R., Sams, M., \& Nummenmaa, L. (2014). Synchronous brain activity across individuals underlies shared psychological perspectives. Neurolmage, 100, 316-324.

Lang, A. (2009). The limited capacity model of motivated mediated message processing. In R. L. Nabi \& M. B. Oliver (Eds.), The Sage handbook of media processes and effects (pp. 99-112). Los Angeles, CA: Sage.

Lang, P. J. (2010). Emotion and motivation: Toward consensus definitions and a common research purpose. Emotion Review, 2, 229-233.

Lang, P. J., Bradley, M. M., \& Cuthbert, B. N. (1997). Motivated attention: Affect, activation, and action. In P. J. Lang, R. F. Simons, \& M. Balaban (Eds.), Attention and orienting; Sensory and motivational processes (pp. 97-135). New York, NY: Routledge.

Lehne, M., Engel, P., Rohrmeier, M., Menninghaus, W., Jacobs, A. M., \& Koelsch, S. (2015). Reading a suspenseful literary text activates brain areas related to social cognition and predictive inference. PLoS One, 10, e0124550.

Lehne, M., \& Koelsch, S. (2015). Toward a general psychological model of tension and suspense. Frontiers in Psychology, 6, 79. https://doi.org/10.3389/fpsyg.2015.00079

Levy, M. R. (1982). The Lazarsfeld-Stanton Program Analyzer: An historical note. Journal of Communication, 32(4), 30-38. https://doi.org/10.1111/j.1460-2466.1982.tb02516.x

McNamara, Q., De la Vega, A., \& Yarkoni, T. (2017). Developing a comprehensive framework for multimodal feature extraction. Proceedings of the 22nd ACM SIGKDD International Conference on Knowledge Discovery and Data Mining. https://doi.org/ 10.1145/3097983.3098075

Menon, V. (2015). Salience network. In A. W. Toga (Ed.), Brain mapping: An encyclopedic reference (Vol. 2, pp. 597-611). Waltham, MA: Academic Press.

Mesulam, M. M. (1998). From sensation to cognition. Brain, 121, 1013-1052.

Naci, L., Cusack, R., Anello, M., \& Owen, A. M. (2014). A common neural code for similar conscious experiences in different individuals. Proceedings of the National Academy of Sciences of the United States of America, 111, 14277-14282.
Najafi, M., Kinnison, J., \& Pessoa, L. (2017). Dynamics of intersubject brain networks during anxious anticipation. Frontiers in Human Neuroscience, 11, 552. https://doi.org/10.3389/ fnhum.2017.00552

Nastase, S. A., Gazzola, V., Hasson, U., \& Keysers, C. (2019). Measuring shared responses across subjects using intersubject correlation. Social Cognitive and Affective Neuroscience, 14, 667-685.

Nummenmaa, L., Glerean, E., Viinikainen, M., Jääskeläinen, I. P. Hari, R., \& Sams, M. (2012). Emotions promote social interaction by synchronizing brain activity across individuals. Proceedings of the National Academy of Sciences of the United States of America, 109, 9599-9604.

Nummenmaa, L., Lahnakoski, J. M., \& Glerean, E. (2018). Sharing the social world via intersubject neural synchronisation. Current Opinion in Psychology, 24, 7-14.

Nummenmaa, L., Saarimäki, H., Glerean, E., Gotsopoulos, A., Jääskeläinen, I. P., Hari, R., \& Sams, M. (2014). Emotional speech synchronizes brains across listeners and engages large-scale dynamic brain networks. Neurolmage, 102, 498-509.

Olsson, A., Nearing, K. I., \& Phelps, E. A. (2007). Learning fears by observing others: The neural systems of social fear transmission. Social Cognitive and Affective Neuroscience, 2, 3-11.

Pei, R., Schmälzle, R., Kranzler, E. C., O'Donnell, M. B., \& Falk, E. B. (2019). Adolescents' neural response to tobacco prevention messages and sharing engagement. American Journal of Preventive Medicine, 56, S40-S48.

Pessoa, L. (2009). How do emotion and motivation direct executive control? Trends in Cognitive Sciences, 13, 160-166. https://doi. org/10.1016/j.tics.2009.01.006

Pessoa, L. (2013). The cognitive-emotional brain: From interactions to integration. Cambridge, MA: MIT Press.

Pessoa, L. (2018). Understanding emotion with brain networks. Current Opinion in Behavioral Sciences, 19, 19-25.

Poldrack, R. A. (2006). Can cognitive processes be inferred from neuroimaging data? Trends in Cognitive Sciences, 10, 59-63.

Potter, R. F., \& Bolls, P. D. (2012). Psychophysiological measurement and meaning: Cognitive and emotional processing of media. New York, NY: Routledge.

Power, J. D., Schlaggar, B. L., \& Petersen, S. E. (2015). Recent progress and outstanding issues in motion correction in resting state fMRI. Neurolmage, 105, 536-551.

Preti, M. G., Bolton, T. A., \& Van De Ville, D. (2017). The dynamic functional connectome: State-of-the-art and perspectives. Neurolmage, 160, 41-54.

Raz, G., \& Hendler, T. (2014). Forking cinematic paths to the self: Neurocinematically informed model of empathy in motion pictures. Projections, 8(2), 89-114.

Richardson, H., Lisandrelli, G., Riobueno-Naylor, A., \& Saxe, R. (2018). Development of the social brain from age three to twelve years. Nature Communications, 9, 1027.

Roy, M., Shohamy, D., \& Wager, T. D. (2012). Ventromedial prefrontal-subcortical systems and the generation of affective meaning. Trends in Cognitive Sciences, 16, 147-156.

Sara, S. J., \& Bouret, S. (2012). Orienting and reorienting: The locus coeruleus mediates cognition through arousal. Neuron, 76, 130141.

Schmälzle, R., \& Grall, C. (in press). Mediated messages and synchronized brains. In R. Weber \& K. Floyd (Eds.), Handbook of Communication Science and Biology. New York, NY: Routledge.

Schmälzle, R., Häcker, F., Honey, C. J., \& Hasson, U. (2015). Engaged listeners: Shared neural processing of powerful political speeches. Social, Cognitive, and Affective Neurosciences, 1, 168-169.

Schmälzle, R., Häcker, F., Renner, B., Honey, C. J., \& Schupp, H. T. (2013). Neural correlates of risk perception during real-life risk communication. The Journal of Neuroscience, 33, 10340-10347. 
Schmälzle, R., Imhof, M. A., Grall, C., Flaisch, T., \& Schupp, H. T. (2017, July 3). Reliability of fMRI time series: Similarity of neural processing during movie viewing. bioRxiv. Retrieved from https://www.biorxiv.org/content/10.1101/158188v1.article-info

Schmitz, T. W., \& Johnson, S. C. (2007). Relevance to self: A brief review and framework of neural systems underlying appraisal. Neuroscience and Biobehavioral Reviews, 31, 585-596.

Schupp, H. T., Flaisch, T., Stockburger, J., \& Junghöfer, M. (2006). Emotion and attention: Event-related brain potential studies. Progress in Brain Research, 156, 31-51.

Schupp, H. T., Kirmse, U., Schmälzle, R., Flaisch, T., \& Renner, B. (2016). Newly-formed emotional memories guide selective attention processes: Evidence from event-related potentials. Scientific Reports, 6, 28091. https://doi.org/10.1038/srep28091

Schupp, H. T., Stockburger, J., Codispoti, M., Junghöfer, M., Weike, A. I., \& Hamm, A. O. (2007). Selective visual attention to emotion. The Journal of Neuroscience, 27, 1082-1089.

Seeley, W. W., Menon, V., Schatzberg, A. F., Keller, J., Glover, G. H., Kenna, H., ... Greicius, M. D. (2007). Dissociable intrinsic connectivity networks for salience processing and executive control. The Journal of Neuroscience, 27, 2349-2356.

Shafto, M. A., Tyler, L. K., Dixon, M., Taylor, J. R., Rowe, J. B., Cusack, R., ... Cam-CAN. (2014). The Cambridge Centre for Ageing and Neuroscience (Cam-CAN) study protocol: A crosssectional, lifespan, multidisciplinary examination of healthy cognitive ageing. BMC Neurology, 14, 204.

Shen, X., Tokoglu, F., Papademetris, X., Constable, R. T. (2013). Groupwise whole-brain parcellation from resting-state fMRI data for network node identification. Neurolmage, 82, 403-415.

Sherif, M., \& Sargent, S. S. (1947). Ego-involvement and the mass media. The Journal of Social Issues, 3, 8-16.

Shirer, W. R., Ryali, S., Rykhlevskaia, E., Menon, V., \& Greicius, M. D. (2012). Decoding subject-driven cognitive states with wholebrain connectivity patterns. Cerebral Cortex, 22, 158-165.

Tamborini, R. (2011). Moral intuition and media entertainment. Journal of Media Psychology, 23, 39-45. https://doi.org/ 10.1027/1864-1105/a000031

Tamborini, R., Bowman, N. D., Eden, A., Grizzard, M., \& Organ, A. (2010). Defining media enjoyment as the satisfaction of intrinsic needs. The Journal of Communication, 60, 758-777.

Taylor, J. R., Williams, N., Cusack, R., Auer, T., Shafto, M. A., Dixon, M., ... Henson, R. N. (2017). The Cambridge Centre for Ageing and Neuroscience (Cam-CAN) data repository: Structural and functional MRI, MEG, and cognitive data from a crosssectional adult lifespan sample. Neurolmage, 144, 262-269.

Turner, B. O., Paul, E. J., Miller, M. B., \& Barbey, A. K. (2018). Small sample sizes reduce the replicability of task-based fMRI studies. Communications Biology, 1, 62.

Vanderwal, T., Eilbott, J., \& Castellanos, F. X. (2019). Movies in the magnet: Naturalistic paradigms in developmental functional neuroimaging. Developmental Cognitive Neuroscience, 36, 100600.

Vorderer, P., Wulff, H. J., \& Friedrichsen, M. (2013). Suspense: Conceptualizations, theoretical analyses, and empirical explorations. Mahwah, NJ: Routledge.

Watt, J. H. (1994). Detection and modeling of time sequenced processes. In A. Lang (Ed.), Measuring psychological responses to media messages (pp. 181-207). Hillsdale, NJ: Erlbaum.

Weber, R., Eden, A., Huskey, R., Mangus, M., \& Falk, E. (2015). Bridging media psychology and cognitive neuroscience. Journal of Media Psychology, 27, 146-156. https://doi.org/10.1027/ 1864-1105/a000163

Yeshurun, Y., Swanson, S., Simony, E., Chen, J., Lazaridi, C., Honey, C. J., \& Hasson, U. (2017). Same story, different story: The neural representation of interpretive frameworks. Psychological Science, 28, 307-319.
Zillmann, D. (1980). Anatomy of suspense. In P. Tannenbaum (Ed.), The entertainment functions of television (pp. 133-163). Hillsdale, NJ: Erlbaum.

Zillmann, D. (1996). The psychology of suspense in dramatic exposition. In P. Vorderer, H. J. Wulff, \& M. Friedrichsen (Eds.), Suspense: Conceptualizations, theoretical analyses, and empirical explorations (pp. 199-231). Mahwah, NJ: Routledge.

\section{History}

Received December 2, 2018

Revision received August 27, 2019

Accepted August 30, 2019

Published online March 9, 2020

\section{Acknowledgments}

We thank the CamCan Consortium for providing the data. We thank three anonymous reviewers for helpful suggestions. We acknowledge the support of the creators of the nilearn, BrainlAK, pandas, seaborn, scipy, and Jupyter software packages, and the high-performance computing cluster at the Institute for CyberEnabled Research (https://icer.msu.edu).

\section{Authorship}

Ralf Schmälzle and Clare Grall contributed equally to this article.

\section{Open Data}

All analyses are documented at https://github.com/nomcomm/ CamCanSuspenselSC_JMP.

\section{ORCID}

Ralf Schmälzle

(D) https://orcid.org/0000-0002-0179-1364

\section{Ralf Schmälzle}

Department of Communication

College of Communication Arts and Sciences

Michigan State University

404 Wilson Rd.

East Lansing, MI 48824

USA

schmaelz@msu.edu

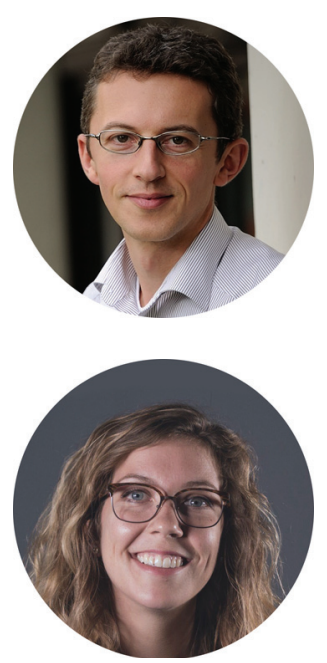

Ralf Schmälzle (PhD, University of Konstanz) is an assistant professor at the Department of Communication, Michigan State University. He is interested in cognitive and affective responses to mass communication, including the reception of movies, speeches, and health messages.

Clare Grall (MA, Michigan State University) is a PhD candidate of the Department of Communication at Michigan State University. She uses biological approaches to study the positive social and emotional effects of narrative engagement. 